\title{
AVALIAÇÃO DO DESTINO E BIOACUMULAÇÃO DE BENZO(A)PIRENO ATRAVÉS DE SIMULAÇÃO COMPUTACIONAL
}

\author{
Sandro Froehner* e Raquel Fernandes Martins
}

Departamento de Engenharia Ambiental, Universidade Federal do Paraná, Centro Politécnico, 81531-990 Curitiba - PR, Brasil

Recebido em 25/4/07; aceito em 11/10/07; publicado na web em 2/4/08

\begin{abstract}
ASSESSMENT OF FATE AND BIOACCUMULATION OF BENZO(A)PIRENE BY COMPUTER MODELING. The objective of this work was to evaluate the environmental distribution of benzo(a)pirene, a polycyclic aromatic hydrocarbon, by the EQC model. The modeling of the contaminant distribution was accomplished by means of the fugacity model applied to a hypothetical scenario constituted by air, water, soil and sediment. The modeling and simulations revealed that the soil is the preferential compartment. We also discuss the implications of the results about fate and ecological risks associated with benzo(a)pirene. We concluded that the emissions of HPAs can not be ignored and bioaccumulation among others risks can be induced.
\end{abstract}

Keywords: bioaccumulation; hydrocarbon polycyclic aromatics; pollution.

\section{INTRODUÇÃO}

A queima da biomassa e de combustíveis fósseis é considerada como a principal fonte de emissão de material particulado no mundo, ${ }^{1}$ além disso, também são emitidos diversos compostos para a atmosfera, sendo que muitos são carcinogênicos e/ou mutanogênicos, como os hidrocarbonetos policíclicos aromáticos (HPAs). Os HPAs caracterizam-se por possuírem dois ou mais anéis aromáticos condensados. São formados durante a combustão incompleta ou pirólise de materiais contendo carbono e hidrogênio. As maiores fontes antropogênicas são queima de combustíveis fósseis e atividades agrícolas, como queimada de florestas, queima de cana-de-açúcar, por exemplo. ${ }^{2}$ Também podem ser encontrados no óleo diesel. ${ }^{3}$

Os HPAs de modo geral são tóxicos, carcinogênicos em muitos animais, atuam como anti-estrogênios em mamíferos e induzem toxicidade reprodutiva em mulheres. ${ }^{4}$ Muitos autores também atribuíram efeitos embriotóxicos e teratogênicos a alguns HPAs. ${ }^{5}$ O comportamento dos HPAs nos meio ambiente depende da pressão de vapor e solubilidade em água. Aqueles que apresentam uma baixa pressão de vapor e são hidrofóbicos têm pouca mobilidade quando atingem o solo, sendo a adsorção um processo importante, tanto em solos, sedimentos e também no material particulado presente na fase gasosa. A biodegradação é o meio mais comum de remoção dos HPAs no meio ambiente, todavia, HPAs parcialmente dissolvidos em água tendem a se adsorverem nos sólidos suspensos e em sedimentos na coluna d'água, fato justificado pelo alto valor de Koc (coeficiente de partição correlacionado com substâncias orgânicas). Devido à baixa solubilidade, os HPAs presentes em corpos d'água podem se acumular em tecidos gordurosos de organismos que compõem a cadeia trófica. ${ }^{6} \mathrm{O}$ fator de bioconcentração (FBC) é dado pela razão da concentração do composto poluente no organismo e a concentração do composto poluente na água. O valor do $\mathrm{FBC}^{7}$ para o benzo(a)pireno $(\mathrm{BaP})$ é aproximadamente de 1 $\mathrm{x} 10^{5}$. As consequiências da tendência bioacumuladora de um composto orgânico podem representar um risco ecológico, sendo que o risco pode significar um processo carcinogênico, interferência na reprodução etc. ${ }^{8} \mathrm{O}$ risco ecológico é, geralmente, definido como o

*e-mail: froehner@ufpr.br quociente de risco, ou seja, pela razão entre a concentração do poluente disponível no meio ambiente e a concentração que causa um efeito adverso. Valores acima de 1 indicam risco em potencial. ${ }^{5}$

O destino e a migração de contaminantes no meio ambiente são controlados pelas propriedades físico-químicas do composto e pela natureza do meio físico para o qual o composto migra, assim como determinam o movimento e a separação. As principais propriedades são a solubilidade, volatilidade e o coeficiente de partição octanolágua, responsável pela tendência bioacumuladora de um composto.

A avaliação do impacto ambiental de compostos orgânicos poluentes e persistentes sobre o ecossistema é essencial para avaliação de riscos ecológicos, avaliação do ciclo de impacto e, sobretudo, na prevenção. ${ }^{5,8}$ A compreensão das interações dos compostos orgânicos com o meio ambiente é essencial na avaliação dos efeitos e impactos causados no meio ambiente, ${ }^{9,10}$ deste modo, torna-se imprescindível a determinação quantitativa dos poluentes nos vários compartimentos possíveis (água, ar, solo e sedimento). Para tal finalidade, existem modelos matemáticos que descrevem com boa precisão e rapidez o destino e as concentrações do poluente em cada compartimento, sendo extremamente importante a avaliação de compostos de alta periculosidade e toxicidade. ${ }^{11}$ Os diferentes impactos dependem da quantidade de poluente emitido para o meio ambiente, das características físico-químicas do poluente, do transporte e mobilidade, do tempo de permanência, bem como da toxicidade do poluente químico para o ecossistema. ${ }^{11,12}$ Assim, num caso de emergência, os modelos para estimar o destino e a concentração de compostos são extremamente úteis, além de identificarem as possíveis partições dos compostos entre os compartimentos e o tempo de permanência. Muitos modelos prevêem a bioacumulação. ${ }^{13}$ Efeitos adversos podem ser resultado da presença de um contaminante em concentrações significativas na água, sedimento, solo ou mesmo no ar. Os efeitos adversos também dependem do tempo de exposição e da concentração.

Modelos matemáticos podem contribuir para prever o destino e a preferência ambiental de poluentes e podem sugerir quais poluentes e em quais compartimentos devem ser sistematicamente investigados em programas de monitoramento.

O objetivo principal deste trabalho foi utilizar o modelo EQC, ${ }^{9}$ recomendado pela Agência de Proteção Ambiental Americana $(\mathrm{USEPA})^{14}$ e pela Organização para Cooperação Econômica e De- 
senvolvimento (OECD), ${ }^{15}$ para avaliar o destino do BaP no meio ambiente emitido para a atmosfera. $\mathrm{O}$ modelo é proposto como uma estratégia de análise da exposição ambiental às substâncias químicas. Com base na fugacidade, o modelo estima a concentração e partição em cada compartimento no meio ambiente. ${ }^{9,16}$ Assim estimou-se a concentração do $\mathrm{BaP}$ em vários compartimentos em diferentes níveis de complexidade oferecidos pelo modelo EQC, sendo o último nível, o III, o mais realístico. O BaP foi escolhido devido à sua toxicidade e por ser um dos HPAs mais abundantes quando da queima da biomassa. A literatura ainda não apresenta dados suficientes que permitissem comparar os dados obtidos através do modelo com dados analíticos, porém os resultados demonstram que a emissão de HPAs, e em especial o BaP, não pode ser desprezada.

\section{PARTE EXPERIMENTAL}

\section{O modelo EQC}

O modelo EQC foi obtido no endereço eletrônico do Centro Canadense de Modelagem Ambiental (CEMN). ${ }^{17}$

O modelo EQC, de Mackay, está fundamentado no conceito de fugacidade, por ser um termo mais compreensivo que o potencial químico. ${ }^{9} \mathrm{O}$ modelo EQC considera quatro compartimentos: ar, com subcompartimentos (aerossol, fase gasosa e total), água (sólidos suspensos, fase aquosa, fase total), solo com seus subcompartimentos (fase gasosa, fase aquosa, fase solo e fase total) e finalmente o sedimento, também com seus subcompartimentos (fase aquosa, fase sólida e fase total).

Os cálculos foram realizados considerando um cenário hipotético com área fixa de $100.000 \mathrm{~km}^{2}$, sendo que aproximadamente $10 \%$ desta área é composta por água. O modelo também utiliza a altura na atmosfera de $1000 \mathrm{~m}, 20 \mathrm{~m}$ a profundidade máxima da água e considera que a deposição no solo se dê até $0,1 \mathrm{~m}$. Já no sedimento, a deposição ocorre até $0,01 \mathrm{~m}$. Deste modo, temos que o volume total no ar é de $100.000 \mathrm{~km}^{3}, 200 \mathrm{~km}^{3}$ na água, $9 \mathrm{~km}^{3}$ no solo e $0,1 \mathrm{~km}^{3}$ no sedimento. Para os cálculos assumiu-se que a estrutura dos compartimentos é homogênea. As equações usadas para descrever partição entre compartimentos, transporte e reações de transformações dos poluentes e os parâmetros fixos usados no modelo estão descritos em Mackay. ${ }^{9}$

Em termos gerais o modelo está fundamentado na capacidade de partição do composto químico entre os vários compartimentos e subcompartimentos, esta capacidade expressa a afinidade que o composto químico tem por cada compartimento. A afinidade ou fugacidade depende de alguns fatores, como pressão de vapor, solubilidade e fração de carbono orgânico nos compartimentos. Por exemplo, para o BaP a afinidade para o compartimento ar $\left(\mathrm{Z}_{\mathrm{ar}}\right)$ é definida como 1/RT, onde R é a constante geral dos gases e T a temperatura em Kelvin. Já para a água, a afinidade $\left(\mathrm{Z}_{\text {agua }}\right)$ é calculada segundo a constante de partição entre ar e água $\left(\mathrm{K}_{\mathrm{AW}}\right)$, este coeficiente pode ser deduzido a partir da pressão de vapor do contaminante. Assim, temos que a partição de um composto entre ar e água é definida pela razão $\mathrm{Z}_{\mathrm{ar}} / \mathrm{K}_{\mathrm{AW}}$. A partição com o solo e o sedimento depende da fração de carbono orgânico em cada compartimento. Para o solo, a afinidade para este compartimento é determinada da seguinte forma $Z_{\text {solo }}=Z_{\text {água }} K_{o c} \cdot \rho_{i} \phi_{i}$. Para o sedimento, a afinidade é definida como $Z_{\text {sed }}=Z_{\mathrm{ar}} \cdot \mathrm{K}_{\mathrm{oc}} \cdot \rho_{\mathrm{i}} \phi_{\mathrm{i}}$, onde $\rho_{\mathrm{i}}$ é a densidade e $\phi_{\mathrm{i}}$ é a fração orgânica do solo e do sedimento. Nos níveis II e III, o modelo considera que haja perdas através de reações de degradação, sendo calculadas através da Equação:

$\mathrm{D}_{\mathrm{Ri}}=\mathrm{V}_{\mathrm{i}} \mathrm{Z}_{\mathrm{i}} \mathrm{k}_{\mathrm{i}}$ onde $\mathrm{V}_{\mathrm{i}}$ é o volume do compartimento em $\mathrm{m}^{3}, \mathrm{Z}_{\mathrm{i}}$ a fugacidade do compartimento, $\mathrm{k}_{\mathrm{i}}$ representa a constante de velocidade para a reação de decomposição $\left(\mathrm{h}^{-1}\right)$. A advecção é considerada apenas para os compartimentos ar e água, sendo calculada segundo a Equação:

$\mathrm{D}_{\mathrm{Ai}}=\mathrm{G}_{\mathrm{i}} \mathrm{Zi}$

em que $\mathrm{G}$ representa fluxo advectivo em cada compartimento (i) $\mathrm{em} \mathrm{m}^{3} / \mathrm{h}$.

O modelo EQC permite o cálculo da distribuição total da massa de um poluente entre compartimentos sendo representado por um sistema linear de equações algébricas e equações diferenciais ordinárias. O modelo permite avaliar o destino e transporte de poluentes em três níveis diferentes, I, II e III. A classificação dos níveis é consequiência da complexidade dos cálculos e das hipóteses envolvidas na formulação de cada nível. Quando todas as fugacidades são iguais e constantes em todos os compartimentos, a distribuição percentual da massa é calculada diretamente, neste caso correspondem ao nível I ou ao nível II, considerando que existam emissões, degradações e advecções do composto em cada compartimento. Já no nível III, o modelo admite que as fugacidades estejam em equilíbrio estacionário e que cada compartimento pode ter diferentes valores de fugacidade. Neste nível é possível ocorrerem degradações, advecções, emissões e transferências. Dependendo das características físico-químicas e propriedades do composto, o poluente é classificado como tipo 1, ou seja, que permite a distribuição em todos os compartimentos. ${ }^{9,10}$ A maioria dos compostos orgânicos são classificados como tipo 1. Compostos que raramente são encontrados no ar são classificados como tipo 2 (cátions, ânions e compostos não voláteis). Compostos muito hidrofóbicos e que dificilmente são encontrados na fase aquosa são classificados como do tipo 3. O BaP é um composto de baixa pressão de vapor, porém encontrado no ar, portanto, classificado como do tipo 1 . Na Tabela 1 estão os valores de entrada requeridos pelo modelo EQC.

Tabela 1. Valores das propriedades físico-químicas do $\mathrm{BaP}$ requeridos pelo modelo EQC

\begin{tabular}{lc}
\hline Propriedade & Valor \\
\hline Peso molecular $(\mathrm{g} / \mathrm{mol})$ & 253,2 \\
Ponto de fusão $\left({ }^{\circ} \mathrm{C}\right)$ & 179 \\
Solubilidade em água $\left(\mathrm{g} / \mathrm{m}^{3}\right)$ & 0,00162 \\
Pressão de vapor $(\mathrm{Pa})$ & $7,32 \times 10^{-7}$ \\
Log K $_{\text {ow }}$ & 6,13 \\
Tempo de meia vida no ar $(\mathrm{h})$ & 170 \\
Tempo de meia vida na água $(\mathrm{h})$ & 1700 \\
Tempo de meia vida no solo $(\mathrm{h})$ & 7416 \\
Tempo de meia vida no sedimento $(\mathrm{h})$ & 8000 \\
\hline
\end{tabular}

\section{Propriedades do benzo(a)pireno}

O benzo(a)pireno, CAS no 50-32-8, também conhecido como 1,4-benzo(a)pireno, é um sólido cristalino pertencente à classe dos hidrocarbonetos poliaromáticos e consta na lista de poluentes primários da USEPA. ${ }^{14} \mathrm{O} \mathrm{BaP}\left(\mathrm{C}_{20} \mathrm{H}_{12}\right)$ tem peso molecular igual a $252,3 \mathrm{~g} / \mathrm{mol}$ e sua solubilidade em água a $25{ }^{\circ} \mathrm{C}$ é igual a $1,6 \mathrm{x}$ $10^{-3} \mathrm{mg} / \mathrm{L}$. Seu ponto de fusão é alto, $179{ }^{\circ} \mathrm{C}$, e sua pressão de vapor é baixa, $5,5 \times 10^{-9} \mathrm{~mm} \mathrm{Hg}$. O BaP é bastante hidrofóbico, sendo que o log da constante de partição octanol-água $\left(\log \mathrm{K}_{\mathrm{ow}}\right)$ é 6,13 , portanto, estando disponível, tem boa possibilidade de bioacumular na cadeia trófica. $\mathrm{O}$ alto valor de $\mathrm{K}_{\mathrm{oc}}$ (constante de 
partição com a fração orgânica do solo) indica pouca mobilidade. $\mathrm{O}$ valor de $\log \mathrm{K}_{\mathrm{oc}}$ é de 6,00. Conclui-se também, a partir dos valores de pressão de vapor e da constante de Henry $\left(\mathrm{K}_{\mathrm{H}}=1,2 \times 10^{-3} \mathrm{~L}\right.$ $\mathrm{atm} / \mathrm{mol}$ ), que a perda por volatilização é insignificante. ${ }^{7,9}$ Estudos mostram que o tempo de meia-vida por biodegradação no solo é de 120 a 309 dias. Toxicologicamente, o BaP é considerado como carcinogênico, mostra efeitos embriotóxicos e teratogênicos. ${ }^{12}$

\section{RESULTADOS E DISCUSSÃO}

Em geral, o destino de poluentes orgânicos é ditado pela solubilidade e pressão de vapor. Muitos poluentes apresentam valores de $\mathrm{K}_{\mathrm{ow}}$ superiores a 1000 , o que demonstra grande afinidade lipofílica. Esta propriedade faz com compostos poluentes sejam pouco degradados e tenham uma grande possibilidade de bioacumulação, seja em organismos aquáticos ou mesmo em vegetais. $^{7}$ A solubilidade em água diminui com o aumento do peso molecular e se tratando de compostos poliaromáticos é de se esperar que grande parte destes compostos tenham como seu destino final o acúmulo no solo ou quando em contato com a água façam parte do sedimento, especialmente quando o valor de $\log \mathrm{K}_{\mathrm{ow}}$ for maior que 7. Assim, é esperado que, devido às características físico-químicas do BaP, seja um composto persistente e retido, em sua maior parte, no solo e no sedimento.

A Figura 1 mostra o diagrama de distribuição do BaP considerando uma situação hipotética em que são emitidos $10.000 \mathrm{~kg} / \mathrm{h}$ deste composto no ar.

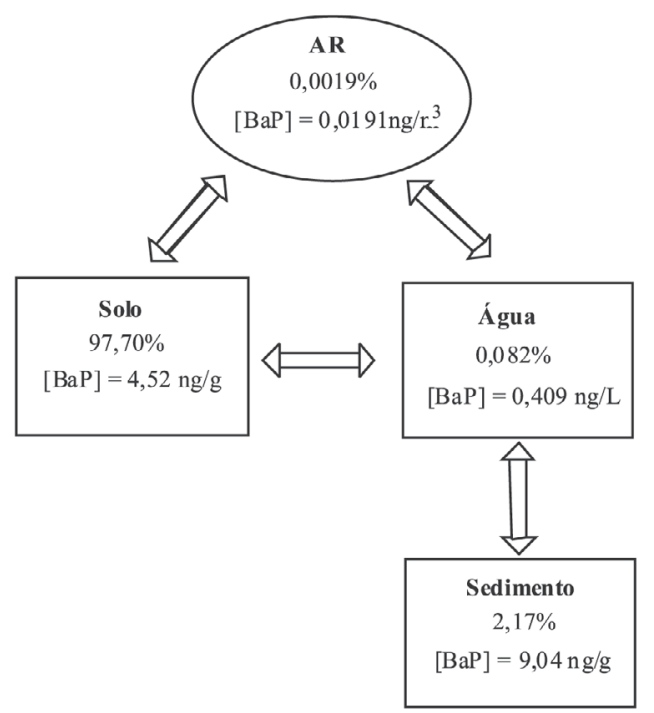

Figura 1. Diagrama de distribuição do benzo(a)pireno entre os compartimentos no nível I

Nesta situação (Nível I) consideramos que a fugacidade em todos os compartimentos é igual. Observamos que o solo retém mais de $97 \%$ do $\mathrm{BaP}$ emitido no ar, enquanto que a quantidade encontrada no ar e na água não ultrapassa $1 \%$. Devemos destacar que não foi considerada a presença de material particulado no ar que pudesse adsorver parte do $\mathrm{BaP}$. No entanto, observa-se que no sedimento encontram-se $2,17 \%$ do $\mathrm{BaP}$ emitido. Considerando a baixa solubilidade do $\mathrm{BaP}$, bem como a sua baixa pressão de vapor, é de se esperar que a maior concentração ocorra no solo e no sedimento. Já no nível II (Figura 2), a complexidade é um pouco maior, pois considera-se que haja os efeitos de advecção e reações.

Os resultados obtidos para esta simulação são semelhantes àqueles obtidos no nível I e, novamente o solo detém a maior parte,

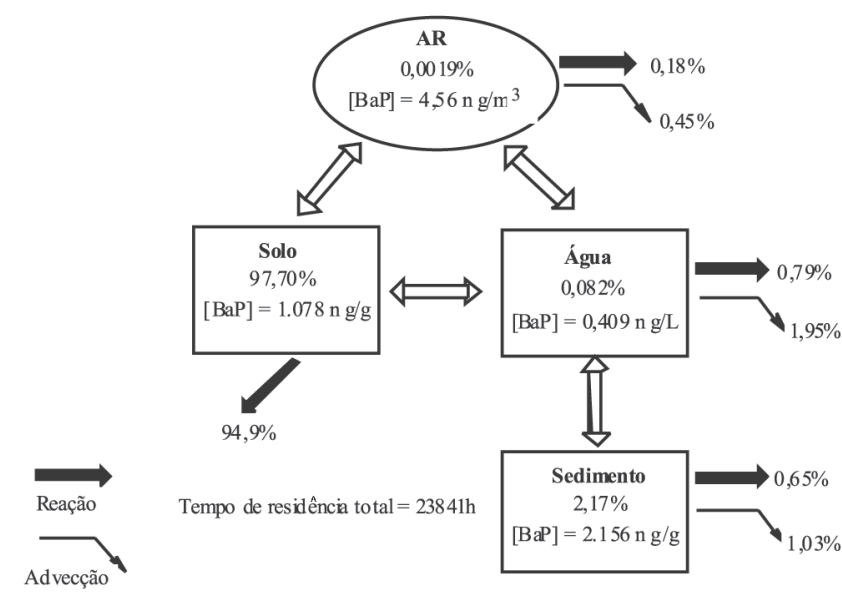

Figura 2. Diagrama de distribuição do benzo(a)pireno entre os compartimentos no nível II

94,9\%. Todavia, ao contrário do nível I, o sistema não está em estado estacionário e observamos mecanismos de remoção, tais como reações no solo que diminuem $57 \%$ da massa total do poluente, a advecção no ar é responsável por $0,45 \%$, já a advecção em água respondeu por $1,95 \%$. A remoção através de reações, tanto na água como no ar, foram 0,79 e $0,18 \%$, respectivamente. Compostos orgânicos como o BaP têm uma baixa solubilidade em água, desta forma justifica-se a maior quantidade encontrada no solo e não no ar, mesmo que a fonte emissora esteja exclusivamente no ar. Esta afinidade com o solo pode representar graves problemas para a vegetação, pois compostos desta natureza podem ser absorvidos pelas plantas através das raízes, além da absorção do composto no ar, o qual pode ser absorvido pelas folhas. ${ }^{18}$

O tempo total, calculado, de permanência deste composto foi de 23.841 h ou 993 dias. A reação predominante ocorre no solo, já que a destruição fotoquímica com a participação de $\mathrm{OH}^{\bullet}$ na troposfera contribui muito pouco, pois a concentração maior está no solo e não no ar. ${ }^{16}$

A Figura 3 mostra os resultados num cenário mais complexo que os casos anteriores, já que neste nível as fugacidades não estão em equilíbrio estacionário e cada compartimento pode ter diferentes valores de fugacidade descrevendo degradação, advecção, emissões e transferências entre compartimentos.

Os valores obtidos referem-se ao compartimento total, portan-

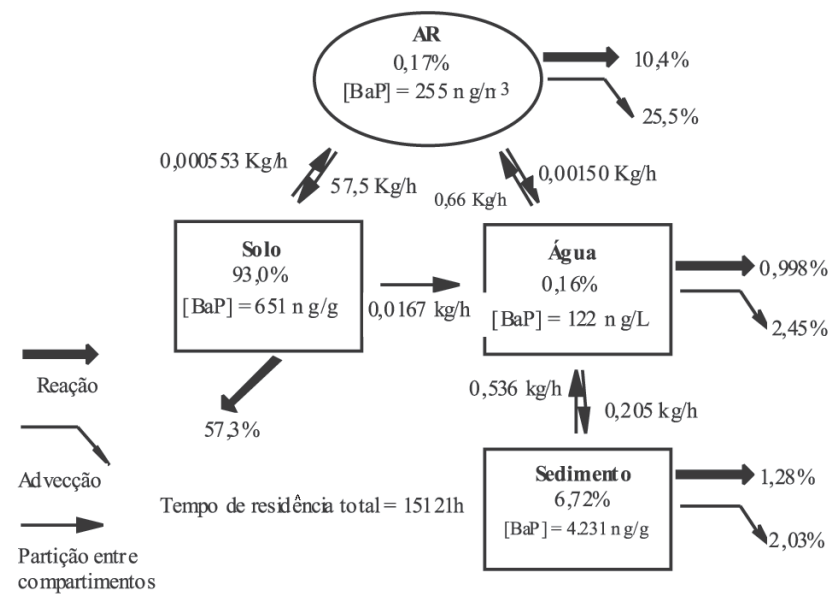

Figura 3. Diagrama de distribuição do benzo(a)pireno entre os compartimentos no nível III 
to, incluem-se subcompartimentos. Os valores obtidos são muito próximos dos valores encontrados nos níveis anteriores, no entanto, observa-se uma maior quantidade do poluente no sedimento, que neste caso foi de $6,72 \%$. Não obstante, o solo ainda retém grande parte da massa de $\mathrm{BaP}$ emitido no ar, $93 \%$. Pequenas quantidades são encontradas na água e no ar, aproximadamente $0,16 \%$ em ambos os casos. As informações adicionais no nível III mostram uma simulação mais realística devido à susceptibilidade do $\mathrm{BaP}$, assim como outros HPAs, no transporte. Segundo Mackay, ${ }^{9}$ para este nível, o cálculo através do modelo deve ser realizado separadamente para cada emissão, ou seja, emissão no ar, na água e no solo e, finalmente, como um todo considerando as três formas de emissão. Neste trabalho estamos apenas considerando a emissão através do ar, pois o interesse maior era a avaliação do comportamento de HPAs lançados no meio ambiente advindos de atividades antropogênicas. Ressalta-se ainda que uma série de transferências entre compartimentos, ar-solo e água-sedimento, são possíveis. A transferência entre solo-água foi desconsiderada, pois devido à baixa solubilidade do $\mathrm{BaP}$ este composto não é lixiviado. $\mathrm{O}$ potencial de lixiviação pode ser determinado pelo índice de Gustafson ${ }^{19}$ e para o $\mathrm{BaP}$ o valor encontrado é menor que 2,8 , ou seja, não lixiviante. No nível III destaca-se a importância da deposição do ar para o solo e a água, cuja taxa excede a taxa de advecção. O tempo de permanência também é longo, aproximadamente 2 anos. A literatura cita como 3 anos o tempo de permanência para o $\mathrm{BaP}^{12}$ A meia vida no solo de poluentes orgânicos aumenta com o aumento da massa molecular, pois a tendência à sorção na matriz sólida do solo é maior. ${ }^{7,20}$ Deste modo, a reação de degradação no solo ainda é a predominante. Parâmetros como transporte e transformação são os responsáveis por processos principais como a degradação, já a deposição e partição água-sedimento respondem pelos processos intermediários. A distribuição do poluente no solo e no sedimento está relacionada com o valor do coeficiente de sorção, que é a partição do contaminante na fração orgânica do solo ou sedimento. No caso do $\mathrm{BaP}$ o valor de $\mathrm{K}_{\mathrm{oc}}$ é 5,54 x 10². A força de sorção no solo ou sedimento depende das propriedades físico-químicas do contaminante e da percentagem de carbono orgânico no material sólido. ${ }^{20}$ Muitos autores concordam que o comportamento de retenção de compostos orgânicos no solo é fortemente dependente da quantidade de matéria orgânica do solo, ${ }^{7,9,19-21}$ sendo o ácido húmico o constituinte da matéria orgânica que mais tende a fixar substâncias pouco solúveis em água.

As concentrações apresentadas nas Figuras 1, 2 e 3 foram obtidas dividindo-se a massa de $\mathrm{BaP}(\mathrm{em} \mathrm{g})$ pelo volume total $\left(\mathrm{m}^{3}\right)$ do compartimento. A concentração na fase sólida $(\mu \mathrm{g} / \mathrm{g})$ é a concentração em $\mathrm{g} / \mathrm{m}^{3}$ dividida pela densidade da fase sólida $\left(2,4 \mathrm{~g} / \mathrm{cm}^{3}\right)$ seca. Assim, a concentração na fase sólida pode ser 1,25 vezes maior e no sedimento, 2,67 vezes maior. ${ }^{16}$

Os resultados mostram que o modelo é capaz de identificar o compartimento dominante e as características de transporte e transferência do contaminante. Como podemos observar, no nível III a concentração na água foi de $0,122 \mu \mathrm{g} / \mathrm{L}$ e no sedimento, $4,231 \mu \mathrm{g} / \mathrm{g}$.

$\mathrm{O}$ quociente de risco ${ }^{5}(\mathrm{QR})$ é dado pela divisão da concentração do composto químico na água (CEA) pela concentração que produz efeito adverso (CTE):

$\mathrm{QR}=\mathrm{CEA} / \mathrm{CTE}$

Para o BaP, a concentração que produz efeito adverso é acima de $0,1 \mu \mathrm{g} / \mathrm{L}$. Considerando que o alvo seja exclusivamente peixe e que esta contaminação possa ser através da assimilação do contaminante através das guelras, o QR encontrado foi de 1,22. Valores de QR maiores que 1 indicam que há riscos de efeitos adversos, sendo a bioacumulação um destes efeitos, além de efeitos como esterilidade ou infertilidade. $\mathrm{O}$ processo de bioacumulação pode ter um papel importante no aumento das concentrações nos organismos. $\mathrm{O}$ BFC em organismos aquáticos ou na biota aquática (BFC) foi estimado pela relação abaixo: ${ }^{7}$

$B F C=10^{[0,76 x \log (K o w)-0,5]}$

Para o BaP, o valor de BFC encontrado foi de 14.414, ou seja numa situação real em que há a emissão de HPAs, a pouca massa de $\mathrm{BaP}$ que tiver a água como destino, a concentração nos tecidos de organismos como peixes poderá ser 14 mil vezes maior que a concentração disponível na água. Segundo Mackay, ${ }^{9}$ poluentes orgânicos prejudiciais à cadeia alimentar que podem bioconcentrar na biota aquática apresentam valores de $\mathrm{K}_{\text {ow }}$ entre $10^{2}$ e $10^{6}$. É importante salientar que este valor de BFC é uma estimativa e que varia com as espécies de peixes, bem como com o hábito alimentar de cada espécie, entretanto, este valor é alto e demonstra claramente que o processo de bioacumulação não pode ser descartado quando ocorrer emissão de compostos poliaromáticos.

As simulações demonstram que pode haver contaminação do solo, água, sedimento e em menor proporção do ar. Devido à baixa solubilidade do $\mathrm{BaP}$, assim como de outros HPAs de maior peso molecular, a mobilidade também é dificultada já que a lixiviação não é favorecida. As perdas dos compostos por degradação microbiológica e fotólise ocorrem em taxas muito baixas e a volatilização também é extremamente pequena devido à baixa pressão de vapor, fator que também contribui para a permanência do composto no meio ambiente.

\section{CONCLUSÃO}

Os resultados mostram que na emissão de HPAs, especialmente do $\mathrm{BaP}$, o principal destino é o solo e os sedimentos, devido à sua baixa solubilidade em água e baixa pressão de vapor. O solo e sedimento são os compartimentos preferenciais do poluente. Porém, no caso de emissão de HPAs para o meio ambiente, não podemos descartar a possibilidade de contaminação de ambientes aquáticos e, devido às características físico-químicas destes compostos, a possibilidade de bioacumulação não pode ser desconsiderada. Os altos valores de $\mathrm{K}_{\text {ow }}$ são fatores determinantes na bioacumulação ao longo da cadeia trófica. Claramente podemos evidenciar que o risco ecológico existe.

A avaliação através do modelo, embora com cenários hipotéticos, é útil na determinação da distribuição ambiental de compostos orgânicos. O modelo é simples e requer valores de parâmetros básicos do composto químico. O aumento da complexidade, nível I para nível III, sistematicamente revela o comportamento e destino do composto químico no meio ambiente, refletindo uma situação mais realística. $\mathrm{O}$ modelo avalia também o destino de compostos não somente do tipo 1 , mas também compostos classificados como do tipo 2 e 3 . O modelo mostrou-se interessante para avaliar o destino e os processos que afetam a distribuição e decomposição no meio ambiente.

\section{AGRADECIMENTOS}

Ao $\mathrm{CNPq}$ pelo suporte financeiro.

\section{REFERÊNCIAS}

1. Santos, C. Y. M.; Azevedo, D. A.; Neto, F. R. A.; Atmos. Environ. 2002, 36, 3009.

2. Magallhães, D.; Vasconcellos, P. de C.; Bruns, R. E.; Quim. Nova 2007, 30,577 . 
3. Froehner, S.; Mazzuco, L.; Corseuil, H.; Resumos do $12^{\circ}$ International Biotechnology Symposium and Exhibition, Santiago, Chile, 2004.

4. http://toxnet.nlm.nih.gov, acessada em Janeiro 2007.

5. Fatorelli, L.; Dissertação de Mestrado, Universidade Federal de Santa Catarina, Brasil, 2005.

6. Gobas, F. A. P. C.; Ecol. Model. 1993, 69, 1.

7. Paraíba, L. C.; Saito, M. L.; Pesq. Agropec. Bras. 2005, 40, 853.

8. Macleod, M.; McKone, T. E.; Foster, K.; Maddalena, R.; Parkenton, T.; Mackay, D.; Environ. Sci. Technol. 2004, 38, 6225.

9. Mackay, D.; Di Guardo, A.; Paterson, S.; Cowan, C.; Environ. Toxicol. Chem. 1996, 15, 1627.

10. Mackay, D.; Gobas, F. A. P. C.; Environ. Toxicol. Chem. 1987, 6, 495.
11. Burkhard, L.; Environ. Toxicol. Chem. 1998, 17, 383.

12. http:/www.ineris.fr, acessada em Janeiro 2007.

13. Mackay, D.; Fraser, A.; Environ. Pollut. 2000, 110, 375.

14. http://www.epa.gov, acessada em Janeiro 2007.

15. http://www.oecd.org, acessada em Janeiro 2007.

16. Achten, C.; Püttmann, W.; Klasmeier, J.; J. Environ. Monit. 2002, 4, 747.

17. http://www.trentu.ca/academic/aminss/envmodel, acessada em Novembro 2006.

18. Trapp, S.; Matthies, M.; Environ. Sci. Technol. 1995, 29, 2333.

19. Gustafson, D. I.; Environ. Toxicol. Chem. 1989, 8, 339.

20. D'Agostinho, A.; Flues, M.; Quim. Nova 2006, 29, 657.

21. Pinto, G. M. F.; Pinto, J. F.; Maciel Filho, R. ; Quim. Nova 2007, 30, 565 